\title{
Intracerebral Hemorrhage in Patients With Chronic Liver Disease
}

\author{
Katsumi HOYA, ${ }^{1}$ Yoshihiro TANAKA, ${ }^{1}$ Takanori UCHIDA, ${ }^{1}$ Issei TAKANO, ${ }^{1}$ \\ Masaya NAGAISHI, ${ }^{1}$ Kazuma KOWATA, ${ }^{1}$ and Akio HYODO ${ }^{1}$ \\ ${ }^{1}$ Department of Neurosurgery, Dokkyo Medical University Koshigaya Hospital, \\ Koshigaya, Saitama
}

\begin{abstract}
The characteristics of intracerebral hemorrhage (ICH) accompanying chronic liver disease (CLD) were investigated in ICH patients hospitalized between 1998 and 2008 divided into the CLD group (55 ICHs in 49 patients) and the idiopathic group without CLD (668 ICHs in 648 patients). The CLD group included a subgroup with liver cirrhosis (LC). Age, sex, history of hypertension, Glasgow Coma Scale (GCS) score on admission, and hematoma locations were reviewed. Outcomes on discharge and causes of inhospital death were also studied. Factors associated with life prognosis in CLD patients were investigated using uni- and multivariate analyses. History of hypertension and deep cerebral hemorrhage were less frequent in the LC subgroup compared to the idiopathic group. Distributions of GCS scores on admission were not significantly different, but incidence of in-hospital death was significantly higher in the CLD group than in the idiopathic group. LC was an independent prognostic factor for CLD patients, but hematoma enlargement was not. Death primarily due to ICH was less frequent in the CLD group than in the idiopathic group. In conclusion, hemostatic disorders seemed to be related to site of hemorrhage, but not to life prognosis in the CLD group. Prognosis was mainly worsened by non-neurological complications.
\end{abstract}

Key words: intracerebral hemorrhage, liver dysfunction, liver cirrhosis, chronic hepatitis, liver disease

\section{Introduction}

Liver cirrhosis (LC) often results in coagulopathy and thrombocytopenia, and causes various hemostatic problems. Some studies have examined the relationships between intracerebral hemorrhage (ICH) and LC, ${ }^{1,6)}$ and abnormalities in laboratory data reflecting liver function reportedly correlate with ICH volume and rebleeding. ${ }^{3,4,11,12)}$ Few investigations have focused on the characteristics of $\mathrm{ICH}$ in patients with chronic liver disease (CLD). This study compared ICH between patients with and without CLD in terms of baseline characteristics, level of consciousness at onset, hematoma location, and prognosis. We also analyzed factors affecting life prognosis in ICH patients with CLD.

\section{Materials and Methods}

We reviewed the hospital records of patients with ICH for the period between April 1998 and September 2008 in the Department of Neurosurgery at Dokkyo Medical University Koshigaya Hospital. This series did not include traumatic ICH patients or those with aneurysmal rupture. We also excluded cases with hemorrhagic complications after cerebral infarction. Potential predisposing factors for ICH were then examined. Patients who had taken antiplatelet drugs or anticoagulants, who suffered any hematological diseases causing thrombocytopenia or coagulopathy, with hemorrhagic cerebrovascular anomalies such as arteriovenous malformation, or who had undergone hemodialysis for chronic renal failure were excluded from this study.

The remaining patients were divided into two

Received April 12, 2011; Accepted September 23, 2011

Author's present address: K. Hoya, MD, Department of Neurosurgery, Teikyo University Chiba Medical Center, Ichihara, Chiba, Japan. 
groups: patients with CLD; and patients without CLD. Patients with CLD were defined as patients who had been diagnosed with chronic hepatitis or LC prior to ICH, or who were diagnosed with such by hepatologists after admission. We included patients who showed abnormal laboratory data for aspartate aminotransferase (>50 IU/l), alanine aminotransferase $(>50 \mathrm{IU} / \mathrm{l})$, or $\gamma$-glutamyl transferase ( $>60 \mathrm{IU} / \mathrm{l}$ ) and with alcoholic consumption of $\geq 400 \mathrm{~g} /$ week for $\geq 10$ years. Alcohol intake was calculated according to the method described previously. ${ }^{8)}$ Patients without any CLD were defined as showing idiopathic ICH. The CLD group included a subgroup with LC diagnosed by a hepatologist.

We reviewed data for age, sex, and presence of hypertension as baseline characteristics. Hypertension was diagnosed if the patient had a history of taking antihypertensive medication or prior diagnosis by a physician. We identified causes for CLD as far as possible, basically according to the diagnosis by hepatologists. The Glasgow Coma Scale (GCS) was used to evaluate neurological severity on admission.

Hematoma locations were classified into the putamen, thalamus, caudate nucleus, cerebellum, brainstem, cerebral lobes, and lateral ventricles. Patients with ICH involving the putamen, thalamus, or caudate were defined as the deep cerebral hemorrhage group. $^{2,9)}$ If hematoma spread over two areas or more, the location was decided according to the site at which hematoma volume was largest on computed tomography (CT). We defined the lateral ventricle type as absence of evident intraparenchymal hematoma, with hematoma apparent only in the ventricles on CT.

Outcomes at discharge were estimated using the Glasgow Outcome Scale (GOS), as the modified Rankin scale had not been used in the earlier years covered by this study. We mainly focused on life prognosis of the patient, in terms of in-hospital death. We assumed that variables representing possible risk factors for death (GOS grade 1) were: age; GCS on admission; deep cerebral hemorrhage; ICH in the posterior fossa; hypertension; alcoholic liver disease; LC; and hematoma enlargement after admission. For the analysis, we classified GCS scores as $<11.5$ or $\geq 11.5$. Hematoma enlargement was defined according to descriptions in the record of the patient. We reviewed the records for in-hospital deaths, identified the cause of death, and judged whether the patient primarily died of $\mathrm{ICH}$, including development of hydrocephalus.

The $\chi^{2}$ test and Student's t test were used for group comparison. Odds ratios and $95 \%$ confidence intervals were obtained using univariate logistic regres- sion in relation to risk factors for in-hospital death. A forward stepwise method was used to construct a multivariate logistic regression model with the inclusion variables from univariate analysis showing values of $p<0.20$. All statistical calculations were performed using SPSS version 16.0 statistical software (SPSS, Chicago, Illinois, USA). Values of $\mathrm{p} \leq 0.05$ were considered statistically significant.

This study was approved by the ethics committee of Dokkyo Medical University and in accordance with the Declaration of Helsinki. Informed consent was obtained from each patient or their authorized guardian.

\section{Results}

A total of 834 ICHs in 803 patients were identified. We excluded 111 hemorrhages in 106 patients from this study because of predisposing factors. The subject population thus comprised 697 patients with 723 ICHs. A total of 55 ICHs in 49 patients with CLD were identified. The LC subgroup comprised 23 patients with 25 ICHs. The remaining 648 patients showed 668 ICHs (idiopathic ICH). Causes of CLD are shown in Fig. 1. About half of the cases in our series were due to hepatitis B or $C$ virus infection.

Age and sex as baseline characteristics were compared between the CLD and idiopathic types, and also between the LC and idiopathic types. No significant differences were found (Table 1). Data on hypertension as a baseline characteristic are also shown in Table 1. We excluded $144 \mathrm{ICH}$ because we could not obtain any information on the episode from the clinical records. The remaining cases were used for analysis. Hypertension was significantly less frequent in CLD patients compared to idiopathic ICH patients $(p<0.001)$. Frequency of hypertension was also significantly lower in LC patients than in idiopathic patients.

Hematoma locations are shown in Fig. 2. Ratios of locations classified into seven categories did not differ significantly between CLD, LC, and idiopathic patients. When locations were classified as deep cerebral hemorrhage or not, the ratio of deep

Table 1 Baseline characteristics of intracerebral hemorrhage patients

\begin{tabular}{llll}
\hline & \multicolumn{1}{c}{ CLD } & \multicolumn{1}{c}{ LC } & \multicolumn{1}{c}{ Idiopathic } \\
\hline Age, mean (SD), yrs & $65.3(8.4)$ & $64.2(7.8)$ & $64.5(12.7)$ \\
Male sex & $33(67.3 \%)$ & $15(65.2 \%)$ & $411(64.4 \%)$ \\
Hypertension & $38(77.6 \%)^{*}$ & $14(63.6 \%)^{*}$ & $492(92.8 \%)$ \\
\hline
\end{tabular}

${ }^{*} \mathrm{p}<0.001$ compared with idiopathic type. CLD: chronic liver disease, LC: liver cirrhosis, SD: standard deviation. 


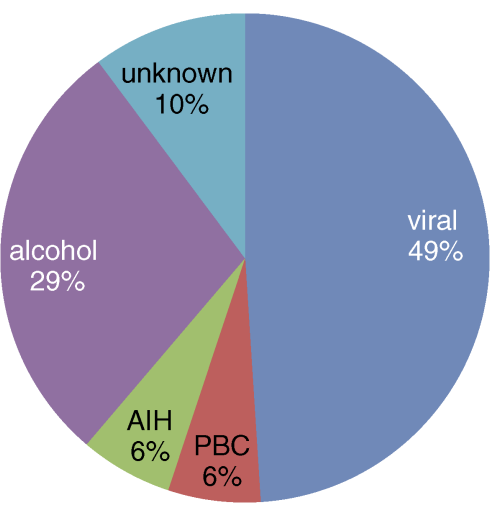

Fig. 1 Causes of chronic liver disease in 49 patients. AIH: autoimmune hepatitis, PBC: primary biliary cirrhosis.

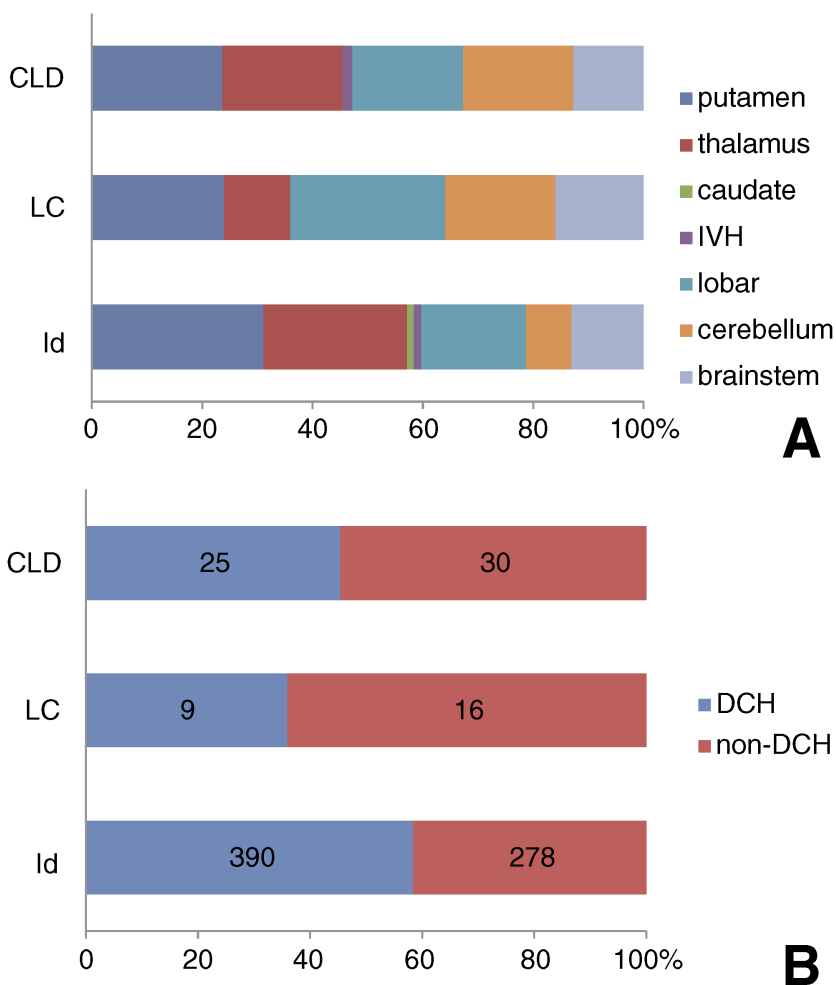

Fig. 2 Hematoma locations in patients with chronic liver disease (CLD), liver cirrhosis (LC), or idiopathic hemorrhage (Id). A: Classification of hematoma locations into seven categories identified no significant differences. IVH: intraventricular hemorrhage. B: Classification of hematoma locations as deep cerebral hemorrhage (DCH) or non-DCH showed that DCH was significantly less frequent in the LC subgroup than in the idiopathic group $(p<0.05)$.

cerebral hemorrhage was significantly lower in the LC group than in the idiopathic ICH group ( $p<$ 0.05).
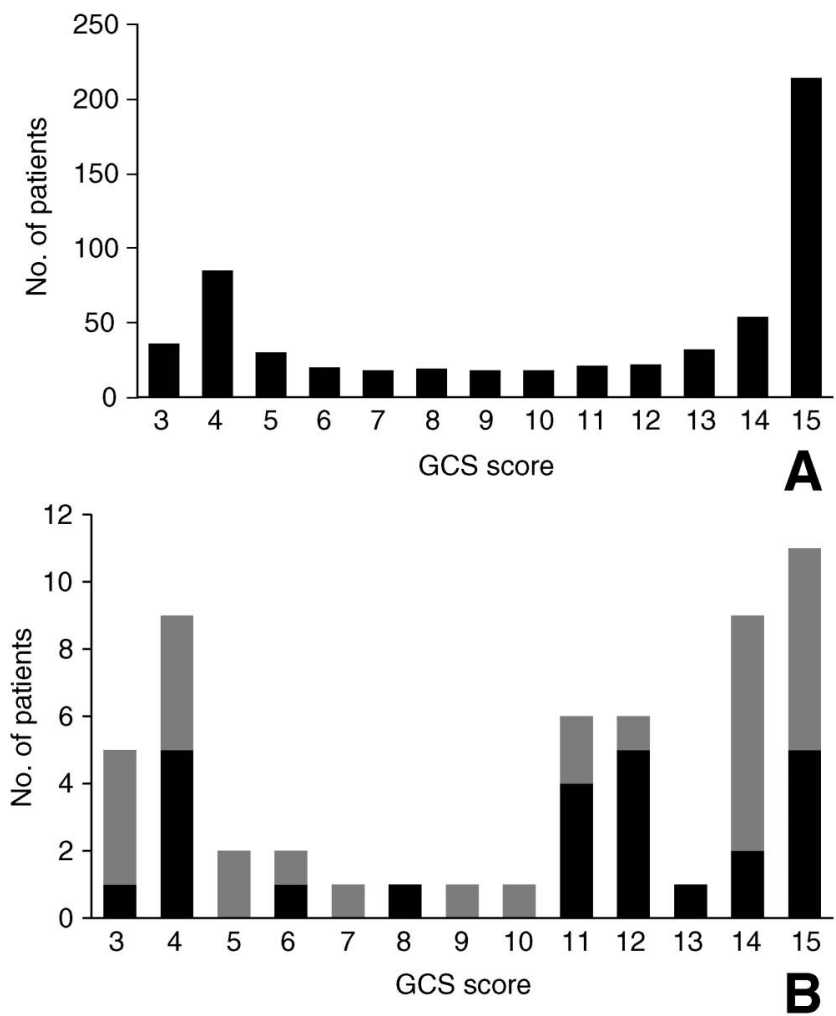

Fig. 3 Distribution of Glasgow Coma Scale (GCS) scores in the idiopathic hemorrhage group (A) and chronic liver disease (CLD) group (B). Black parts in bars of the CLD graph (B) indicate patients with liver cirrhosis. No significant differences were apparent between groups.

Figure 3 shows the distribution of GCS scores for each group. Median GCS scores in the CLD, LC, and idiopathic groups were 11.5, 12, and 13, respectively. No significant differences were apparent. Ratios of patients with GCS score $<11.5$ also showed no significant difference.

When outcomes on discharge were classified as death (GOS grade1) or not, ratio of death was significantly larger in the CLD and LC groups than in the idiopathic ICH group ( $p<0.001$; Fig. 4 A). Causes of in-hospital death were reviewed in the clinical records. The ratio of death primarily due to ICH was very high $(100 / 119)$ in the idiopathic ICH group. Conversely, ratios in the CLD group and LC subgroup were 13/22 and 7/13, respectively (Fig. 4B). Significant differences thus existed between idiopathic ICH and CLD, and between idiopathic ICH and LC ( $p<0.05)$. Among CLD patients, only 1 patient died due to hematoma enlargement. Nine of the CLD group died from non-neurological complications, including disseminated intravascular coagulopathy or multiple organ failure triggered by 
infection in 3 patients, acute respiratory failure due to pneumonia or lung edema in 4 , acute circulatory failure in a patient with massive ascites, and sudden death in a patient with severe hepatic failure.

Table 2 shows the results of univariate and multivariate logistic analyses of relationships between the selected variables and in-hospital death for ICH patients with CLD. Only GCS score $<11.5$ and ICH in the posterior fossa showed significant relation-
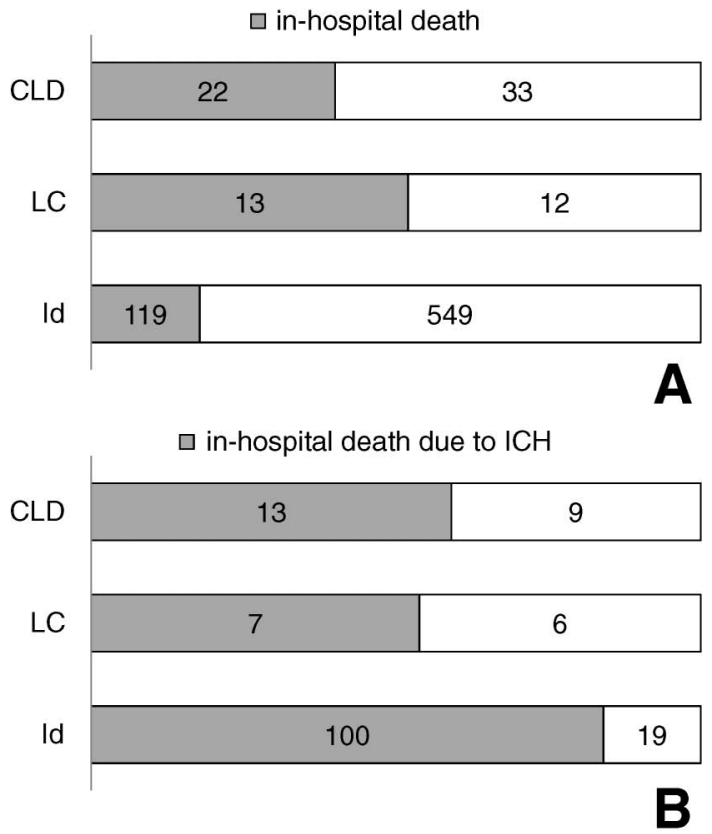

Fig. 4 A: Ratios of patients with in-hospital death. Inhospital deaths were significantly more frequent in the chronic liver disease (CLD) group and the liver cirrhosis (LC) subgroup than in the idiopathic hemorrhage (Id) group (p < 0.001). B: Ratios of in-hospital death due to intracerebral hemorrhage (ICH) among all in-hospital deaths. Significant differences were apparent between Id and CLD, and between Id and LC ( $p<0.05)$. ships to in-hospital death in univariate analysis. For multivariate logistic analysis, we selected 4 variables: GCS <11.5; age; LC; and ICH in the posterior fossa. GCS $<11.5$, age, and LC were identified as independent predictors of in-hospital death in CLD patients (Table 2).

\section{Discussion}

ICH is more frequently found in patients with liver dysfunction than other types of stroke. ${ }^{1,3)}$ However, epidemiological data concerning the incidence of ICH among patients with liver dysfunction compared to the general population appear to be lacking. In our series, hypertension was less frequent in the CLD group and LC subgroup than in the idiopathic ICH group. This may suggest that patients with liver dysfunction are more vulnerable to developing ICH than those without such background pathology.

Some reports have noted that ICH locations in patients with specific hemostatic problems differ from those in patients with general hypertensive ICH. ICH associated with anti-coagulant administration is frequently located in the cerebellum, ${ }^{2,10)}$ whereas the incidence of lobar ICH is high in patients with thrombocytopenia. ${ }^{5)}$ Conversely, hypertension in general is reportedly associated with deep cerebral hemorrhage..$^{7,9)}$ In our study, the incidence of non-deep cerebral hemorrhage, which includes cerebellar and lobar ICH, was significantly higher in the LC subgroup than in the idiopathic ICH group. We suspect that hemostatic disorders are involved in the site of bleeding among LC patients.

Hematoma volumes reportedly increase with greater abnormality of laboratory data for liver function. ${ }^{3,11)}$ We did not measure hematoma volumes, instead estimating neurological severity using GCS on admission. The result showed no significant correla-

Table 2 Uni- and multivariate associations of clinical variables with in-hospital death among chronic liver disease patients

\begin{tabular}{|c|c|c|c|c|c|c|}
\hline & \multicolumn{3}{|c|}{ Univariate associations } & \multicolumn{3}{|c|}{ Multivariate associations } \\
\hline & OR & $95 \% \mathrm{CI}$ & $\mathrm{p}$ Value & OR & $95 \% \mathrm{CI}$ & $\mathrm{p}$ Value \\
\hline Age & 1.049 & $0.98-1.123$ & 0.164 & 1.094 & $1.002-1.194$ & 0.044 \\
\hline GCS $<11.5$ on admission & 10.35 & $2.783-38.488$ & $<0.001$ & 18.1 & $3.228-101.6$ & 0.001 \\
\hline Deep cerebral hemorrhage & 1.000 & $0.338-2.955$ & 1.000 & - & - & - \\
\hline $\mathrm{ICH}$ in the posterior fossa & 3.714 & $1.14-12.101$ & 0.029 & 4.472 & $0.905-22.088$ & 0.066 \\
\hline Hypertension & 1.021 & $0.253-4.115$ & 0.977 & - & - & - \\
\hline Alcoholic liver disease & 1.172 & $0.342-4.011$ & 0.801 & - & - & - \\
\hline $\mathrm{LC}$ & 2.528 & $0.836-7.647$ & 0.101 & 7.398 & $1.318-41.511$ & 0.023 \\
\hline Hematoma enlargement & 2.679 & $0.482-14.893$ & 0.26 & - & - & - \\
\hline
\end{tabular}

CI: confidence interval, GCS: Glasgow Coma Scale, ICH: intracerebral hemorrhage, LC: liver cirrhosis, OR: odds ratio. 
tion with liver dysfunction. Liver dysfunction including hemostatic dysfunction may not be as important in determining the level of consciousness at the onset.

Prognosis reportedly becomes worse with worsening laboratory data for liver functions. ${ }^{3)}$ The severity of LC is also associated with patient outcomes. ${ }^{6}$ Our study showed significantly increased in-hospital deaths for both CLD and LC compared to idiopathic ICH. Considering that GCS on admission in CLD patients was not significantly different from that of idiopathic ICH patients, we speculate that patients with CLD tend to deteriorate after admission and subsequently show poorer prognosis than idiopathic ICH patients. We postulate two reasons for this: hematoma enlargement after admission; and development of complications not directly related to ICH. Six of 117 patients with ICH experienced rebleeding. ${ }^{12)}$ All six showed liver dysfunction, and just two displayed clinical worsening. In our series, only 1 patient in the CLD group died due to hematoma enlargement. Hematoma enlargement was not a significant predictor of in-hospital death in our univariate analysis. We do not think that hematoma enlargement or rebleeding are major factors predicting worsening condition in patients with CLD.

Our multivariate analysis showed LC, along with age and GCS score on admission, as independent predictive factors for in-hospital death in CLD patients. LC is associated not only with hemostatic disorders, but also with other various types of disorders, such as pathologies involving the immunological and endocrinological systems. The ratio of in-hospital deaths unrelated to ICH was significantly higher in the CLD group and LC subgroup compared to the idiopathic ICH group in our study. Nonneurological complications triggered and aggravated by liver dysfunction are important factors in determining the life prognosis of CLD patients.

Blood pressure was intensively controlled in both ICH patients with CLD and ICH patients with idiopathic ICH. Only three LC patients showing severe thrombocytopenia and elongation of prothrombin time underwent platelet and/or frozen fresh plasma transfusion. We do not think such hemostatic treatments are always necessary, but accurate evaluation of liver functions is indispensable to prevent hematoma enlargement. Patients with CLD are susceptible to various types of disorders. Cooperation with hepatologists and other medical staff is important when treating ICH accompanied by CLD.

This retrospective study shows that deep cerebral hemorrhage is less frequent in LC patients. Hemostatic disorders may be involved in determining hematoma locations in such patients. Liver dys- function was not correlated with level of consciousness on admission, but with outcomes on discharge. We think that this was mainly because patients with CLD are at greater risk of non-neurological complications than idiopathic ICH patients.

\section{References}

1) Boudouresques G, Hauw JJ, Meininger V, Escourolle R, Pertuiset B, Buge A, Lhermitte F, Castaigne P: Hepatic cirrhosis and intracranial hemorrhage: significance of the association in 53 pathological cases. Ann Neurol 8: 204-205, 1980

2) Flaherty ML, Haverbusch M, Sekar P, Kissela BM, Kleindorfer D, Moomaw CJ, Broderick JP, Woo D: Location and outcome of anticoagulant-associated intracerebral hemorrhage. Neurocrit Care 5: 197-201, 2006

3) Fujii Y, Takeuchi S, Tanaka R, Koike T, Sasaki O, Minakawa T: Liver dysfunction in spontaneous intracerebral hemorrhage. Neurosurgery 35: 592-596, 1994

4) Fujii Y, Tanaka R, Takeuchi S, Koike T, Minakawa T, Sasaki O: Hematoma enlargement in spontaneous intracerebral hemorrhage. J Neurosurg 80: 51-57, 1994

5) Gasparetto EL, Benites Filho PR, Davaus T, Carvalho Neto A: Central nervous system hemorrhage in thrombocytopenic patients: computed tomographic findings in 21 cases. Arq Neuropsiquiatr 65: 268-272, 2007

6) Huang $\mathrm{HH}$, Lin $\mathrm{HH}$, Shih YL, Chen PJ, Chang WK, Chu HC, Chao YC, Hsieh TY: Spontaneous intracranial hemorrhage in cirrhotic patients. Clin Neurol Neurosurg 110: 253-258, 2008

7) Inagawa $\mathrm{T}$ : Risk factors for primary intracerebral hemorrhage in patients in Izumo City, Japan. Neurosurg Rev 30: 225-234, 2007

8) Iso $\mathrm{H}$, Baba S, Mannami T, Sasaki S, Okada K, Konishi M, Tsugane S; JPHC Study Group: Alcohol consumption and risk of stroke among middle-aged men: the JPHC Study Cohort I. Stroke 35: 1124-1129, 2004

9) Jackson CA, Sudlow CL: Is hypertension a more frequent risk factor for deep than for lobar supratentorial intracerebral haemorrhage? J Neurol Neurosurg Psychiatry 77: 1244-1252, 2006

10) Kase CS, Robinson RK, Stein RW, DeWitt LD, Hier DB, Harp DL, Williams JP, Caplan LR, Mohr JP: Anticoagulant-related intracerebral hemorrhage. Neurology 35: 943-948, 1985

11) Niizuma H, Shimizu Y, Nakasato N, Jokura H, Suzuki J: Influence of liver dysfunction on volume of putaminal hemorrhage. Stroke 19: 987-990, 1988

12) Niizuma H, Suzuki J, Yonemitsu T, Otsuki T: Spontaneous intracerebral hemorrhage and liver dysfunction. Stroke 19: 852-856, 1988

Address reprint requests to: Katsumi Hoya, MD, Department of Neurosurgery, Teikyo University Chiba Medical Center, 3426-3 Anesaki, Ichihara, Chiba 299-0111, Japan.

e-mail: khoya@med.teikyo-u.ac.jp 